\title{
A photoemission study of Pd ultrathin films on $\mathrm{Pt}$ (111)
}

\author{
Bongjin Simon Mun ${ }^{1,2}$, Choongman Lee ${ }^{3}$, Vojislav Stamenkovic ${ }^{1}$, \\ Nenad M. Markovic ${ }^{1}$, and Philip N. Ross Jr. ${ }^{1}$ \\ ${ }^{1}$ Materials Sciences Division, Lawrence Berkeley National Laboratory \\ Berkeley, CA 94720, USA \\ ${ }^{2}$ Advanced Light Source, Lawrence Berkeley National Laboratory \\ Berkeley, CA 94720, USA \\ ${ }^{3}$ Semiconductor R\&D center, Samsung Electronics Co.,LTD, Korea
}

\begin{abstract}
The origin of surface core-level shift (SCLS) of Pd thin films on Pt (111) substrate is investigated. At sub-monolayer coverage of Pd thin films, the splitting of $\mathrm{Pd} 3 d$ core level peaks indicate the contribution of both initial and final-state of photo-ionization processes while no change on valence band (VB) spectra is found. When the coverage of $\mathrm{Pd}$ reaches to single monolayer, the final-state relaxation effect on the $\mathrm{Pd} 3 d$ vanishes and only the initial-state effect, a negative SCLS, is present. Also, the VB spectrum at Pd monolayer films shows a clear band narrowing, that is the origin of the negative SCLS at monolayer coverage. As the Pd coverage is increased to more than monolayer thickness, the $\operatorname{Pd} 3 d$ peaks start to show the surface layer contribution from second and third layers and the VB spectra show even narrower band width, possibly due to the formation of surface states and strained effect of Pd adlayers on top of the first pseudomorphic layer.
\end{abstract}




\section{Introduction}

From the many years of studies on chemical properties of the bimetallic surfaces or alloys, x-ray photoemission spectroscopy (XPS) and ultraviolet photoemission spectroscopy (UPS) measurement have been proved to be powerful sources to provide not only the detailed element-specific chemical information, but also the electronic structures of reacting metal surfaces $\left[{ }^{1},{ }^{2}\right]$. While core-level spectra from XPS can distinguish various chemical properties on the surface from the analysis of surface corelevel shift, the density of state (DOS) measurement from UPS can provide the detailed information of electronic structures from valence band (VB) spectrum $\left[^{3}\right]$. However, due to the complexity of photo-ionization process, careful attentions need to be paid when it comes to interpret subtle changes from the measured spectrum and assign the peak positions to certain physical and chemical origin. Especially, an accurate interpretation on surface core-level shift (SCLS) from XPS spectrum is essential to understanding on structural and chemical information on participating elements. In general, to explain the negative SCLS, lower binding energy, of deposited metal films on various foreign metal substrates, the idea of initial-state band narrowing effect from the reduced-dimensionality of surface layer has been suggested and confirmed in many experiments $\left[{ }^{4}\right]$. At the same time, the presence of final state relaxation mechanism is also found when the deposited metal forms only fractional coverage on surface, resulting in positive SCLS $\left.{ }^{5}\right]$, higher binding energy. 
Palladium has been one of the most researched materials in the world of surface science due to mainly its pronounced reactivity in surface electrochemical and catalytic studies $\left[{ }^{6}\right]$. Consequently, many model studies of Pd and the Pd alloy surface have been performed in ultra-high vacuum environment, and XPS/UPS have been among the most popular measurements for collecting chemical and physical information on the reacting surface. Previously, Wieckowski et al. $\left[^{7}\right]$ has observed the negative SCLS of Pd $3 d$ peaks of Pd thin films on Pt (111) single crystal, and explained the observed negative SCLS with initial-state band narrowing effect. They also concluded that a strong hybridization between Pd thin films and Pt substrate created an efficient screening upon the photo-ionization process, resulting in little final state screening effect. The VB spectrum was not measured in their work.

In this paper, SCLS of Pd thin films on Pt (111) substrate is measured with VB spectrum simultaneously. At sub-monolayer coverage of Pd adlayer, a clear splitting of the Pd $3 d$ core-level spectra is found, and the resulted peak positions are interpreted as initial and final states effects from photo-ionization process of Pd on Pt substrate. The presence of final-state effect of Pd on Pt surface is not observed before. When the Pd coverage reaches to single monolayer (ML), Pd $3 d$ spectra has only single peak at lower binding energy direction, negative SCLS. This negative SCLS, which comes from initialstate band narrowing effect, is also clearly found at the measured VB spectrum. In addition, at monolayer coverage of Pd thin film, the characteristics of VB spectra from UPS show little changes from the DOS of Pt (111) surface, suggesting the deposited Pd layer modifies its electronic structures to the one of Pt surface. As the Pd thin films are 
increased to two or three ML, the $\operatorname{Pd} 3 d$ peaks start to show the surface layer contribution from second and third layers, seen at higher binding energy side. The DOS measurement at this higher coverage shows even narrower VB width, suggesting possible formations of two-dimensional surface states from second and third layers on top of the Pd first ML and the extension of strained effect from the first pseudomorphic ML.

\section{Experiment}

The entire photoemission measurement is carried out at Advance Material Chamber, located at bending magnet beamline 9.3.2. of Advanced Light Source in Lawrence Berkeley National Laboratory. The details of beamline specification can be found in elsewhere $\left.{ }^{8}\right]$. Clean surface of $\mathrm{Pt}(111)$ single crystal is achieved with repeated cycles of Argon sputtering, light annealing at $\mathrm{O}_{2}$ pressure of $\sim 1 \times 10^{-6}$ torr, and a brief UHV annealing at the temperature of $1000 \mathrm{~K}$. The cleaning cycles are repeated until signals of $\mathrm{C}$ and $\mathrm{O}$ are not found from XPS. Upon the cleaning cycle, the ordered surface structure is checked with low energy electron diffraction (LEED) method, generating a clear (1x1) 6-folds hexagonal pattern. The Pd thin films are prepared with thermal evaporation method. The deposition rate of Pd thin film was set to one monolayer (ML) per 7 minutes, which was calibrated with applying quartz crystal microbalance measurement and intensities of XPS core level spectra. During the deposition of Pd film, the chamber pressure was kept under $\sim 5 \times 10^{-10}$ torr. and sample temperature was at room temperature. Scienta hemispherical electron analyzer (SES100) was used to obtain the XPS/UPS measurement and total energy resolution of spectra, including photon energy, 
was set to less than $\sim 0.1 \mathrm{eV}$. The acceptance angle for incoming electrons is $( \pm) 5^{\circ}$ degree. All experimental data were taken under the pressure of $2 \times 10^{-10}$ torr or less.

\section{Results and Discussion}

In Fig. 1(a) and (b), the Pt $4 f_{5 / 2}$ and Pd $3 d_{5 / 2}$ core-level spectra of XPS are shown as the Pd adlayer is increased from 0.2 ML up to 3.0 ML. The photon excitation energy is set to $450 \mathrm{eV}$. From Fig. 1(a), surface states of Pt is clearly seen at lower binding energy (B.E.) side ( $\sim 0.4 \mathrm{eV}$ below the Pt bulk B.E. position) and this surface state contribution disappears as the coverage of Pd reaches to $1.0 \mathrm{ML}$, indicating Pt surface is fully covered with Pd adlayer. On the other hand, the position of bulk Pt $4 f_{5 / 2}$ peak remains constant throughout all cases at binding energy of $70.9 \mathrm{eV}$. In Fig. 1(b), at 0.2 0.4 ML coverage of $\mathrm{Pd}$, it is clear that Pd spectra are composed of at least two different peaks. As the thickness of Pd is increased to $1.0 \mathrm{ML}$, the contribution from the peak at higher binding energy side, i.e. positive SCLS, become small and the peak at lower binding energy side, i.e. negative SCLS, forms a dominant sharp peak. The magnitude of the splitting between those two peaks at sub-monolayer coverage is almost $\sim 0.35 \mathrm{eV}$. As mentioned in introduction, this negative SCLS of Pd thin layer on Pt surface is reported previously and the initial-state band narrowing effect is applied to explain the observed shift $\left[^{7}\right]$. However, the positive SCLS has not been observed at that time and the idea of an efficient screening of Pd core-hole from the strong hybridization between the Pd adlayer and Pt substrate was suggested. On the other hand, the appearance of the positive SCLS of Pd film has been observed when the clusters of Pd 
are prepared on carbon substrates $\left[{ }^{5}\right]$. From the Harsdorff's work $\left[{ }^{5}\right]$, the positive SCLS shift is understood as a lack of efficient final-state screening from substrate. In our experiment, for the first time, both positive and negative SCLS of Pd thin layers in Pt (111) substrate are clearly shown, indicating the presence of both initial and final-state effects from photo-ionization process at sub-monolayer coverage. The positive SCLS indicates the formation of Pd clusters on the surface at a fractional coverage, resulting in final states effects from the photo-ionization process. The cluster formation can possibly prevent immediate screening effect from the Pt substrate. Once the Pd thickness reaches to 2.0 ML, the appearance of extra peak from higher binding energy side can be seen and its intensity is increased further up to $3.0 \mathrm{ML}$, moved toward to bulk value of $\mathrm{Pd} 3 d{ }_{5 / 2}$ peak, $334.9 \mathrm{eV}$. When the Pd coverage reaches 3.0 ML, there are a Pd interface layer, a Pd "bulk layer", and a Pd surface layer. In Fig. 1 (b), there are indicated two arrows on the 3.0 ML Pd spectra. The high binding energy peak represents the surface peak and the other arrow indicates contributions from the subsurface layers, namely the above mentioned "bulk layer" and the interface layer. In addition, it is well known that Pd films on Pt substrate first forms a pseudomorphic layer on the surface and then creates twodimension islands on first adlayer, comparable to Stranski-Krastanov growth mode $\left[{ }^{9},{ }^{10}\right]$.

As a logical step to investigate further on initial-state band narrowing effect, the VB spectra are measured simultaneously. In Fig. 2, the VB spectra are shown as the thickness of Pd thin films is increased. The photon energy of $90 \mathrm{eV}$ is used. Several photon energies are tested and the fine features of VB spectrum are more pronounced when the photon energy of $90 \mathrm{eV}$ is applied. From the Fig. 2, it is rather difficult to find 
any significant changes in the VB spectra at sub-monolayer coverage of Pd thin films. All the main features from clean Pt surface remain the same. As the coverage of Pd adlayer is increased to $1.0 \mathrm{ML}$, the small feature at $5.3 \mathrm{eV}$ from clean Pt surface starts to disappear and the trace of the feature is completely vanished at 3.0 ML coverage. However, all the main peaks and its shape of entire VB spectra of Pd adlayers do not show any noticeable changes from clean Pt VB specturm as Pd coverage is increased up to 3.0 ML. This finding is rather unusual when it is compared to the changes of DOS from the Pd adlayers on different metal substrates. In the case of Pd thin films deposited on Re (0001) surface $\left[{ }^{11}\right]$, the DOS near Fermi level drops significantly and several unique surface features from Pd thin films are shown from VB spectrum. This little changes in the VB spectrum from Pd adlayers on Pt substrate suggest that deposited Pd metals modifies its electronic structures to the one of Pt (111) surface, implying unique, yet significant, changes of Pd characteristic from the bulk Pd metal.

Since the one of the motivations of behind the VB measurement lies on identifying the initial-state band-narrowing effect and its relation to SCLS, the center and width of VB spectrum are calculated $\left[{ }^{12}\right]$. In Fig. 3 , the center and width of measured VB spectra are plotted as a function of Pd coverage on Pt surface. The trend of $d$-band center is almost identical to that of $\mathrm{VB}$ width throughout the all coverage. From the Fig.3, at sub-monolayer coverage (up to $0.6 \mathrm{ML}$ ), the width of VB remains almost the same, showing little changes in the VB spectrum. Then, once the Pd coverage reached to 1.0 ML, a clear initial-state band narrowing effect can be seen. However, the width of the VB keeps on decreasing even after monolayer coverage of Pd. According to the picture 
of initial-state band narrowing effect from surface states, the width of surface VB should reflect the degrees of localization of electron. In other words, the width of VB should be narrowest at a first ML coverage and should start to increase as the thin films forms multilayer structures. However, the trend from Fig. 3 shows exactly the opposite case. The VB gets narrower as the Pd layer is increased even further than single ML.

Followings are the suggested explanation to our experimental result. First, as mentioned previously, throughout all Pd coverage in Fig. 2, it can be seen that the presence of Pd adlayer do not add any significant features on DOS of Pt. In fact, it even looks like the Pd adlayer follows the DOS of Pt substrate, which suggests the presence of unique hybridization between Pd adlayer and Pt substrate. The pronounced reactivity of Pd single ML on Pt surface in the electrochemical and catalytic studies can be possibly due to from this unique, modified electronic structure of $\mathrm{Pd}\left[{ }^{6}\right]$. Secondly, the reduced width of VB at monolayer coverage of Pd can be explained with band narrowing effect from (a) the formation of ideal pseudomorphic Pd layer on top of Pt surface, creating localized reduced-dimensionality of surface layer, (b) the lattice mismatch between the Pd thin films and Pt substrate, i.e Pt $(\sim 2.78 \AA)$ and $\operatorname{Pd}(\sim 2.75 \AA)$. In simple pictures of tight-binding method, the width of VB gets narrow as the lattice distance is increased, due to less overlap of band $\left[{ }^{13}\right]$. In current system, the lattice of pseudomorphic Pd adlayer is stretched upon the lattice of Pt substrate, and this stretched lattice can be the origin of the narrow band width from the VB spectrum in Fig. 3. In fact, it has been well known that the strain effect between adlayer and substrate, caused from the lattice mismatch, can generate unique chemical properties in electrochemical study $\left[{ }^{14}\right]$. Finally, 
the even smaller band width at higher coverage of Pd can be explained with the formation of surface state from second or third layers on top of the first pseudomorphic layer of Pd films, in addition to the extension of stained effect from the first monolayer. As mentioned previously, the surface state causes the narrow band width due to reduced dimensionality and the formation of surface state can be possible even at small coverage of layer.

\section{Conclusion}

The SCLS of Pd thin films on Pt (111) surface has been carefully studied. At submonolayer coverage of Pd, for the first time, both final-state and initial-state effects are observed from Pd $3 d$ core level XPS spectrum. At single ML coverage of Pd, the band narrowing effect, which is originated from a) the formation of ideal two-dimensional Pd surface layer, b) the small lattice mismatch between Pd layer from Pt substrate, is clearly seen from VB spectrum. Also, Pd $3 d$ core-level spectrum shows a sharp negative SCLS at single ML coverage of Pd, which is directly related to initial state band narrowing effect. The measured all VB spectra show no significant changes from the Pt VB spectrum, suggesting the unique hybridization between Pd adlayer and Pt substrate. The narrower band width at higher coverage of Pd is understood with formation of surface states from second and third layers on first Pd pseudomorphic ML coverage on Pt surface and extension of stained effect from the first ML. 


\section{Acknowledgements}

BSM would like to appreciate the valuable discussion with Prof. Charles Fadley and Dr.

Hoon Koh. This research was supported by the Office of Basic Energy Sciences, Materials Sciences Division, of the U.S. Department of Energy under contract DE-AC0376SF00098. 


\section{Figure captions.}

\section{Fig. 1 (a) \& (b)}

The Pt $4 f_{7 / 2}$ and $\mathrm{Pd} 3 d_{5 / 2}$ core level spectra are shown as a function of $\mathrm{Pd}$ coverage on $\mathrm{Pt}$ (111) surface. The photon energy $(h v)$ is set to $450 \mathrm{eV}$ and electron detection angle in XPS is set to normal to the surface.

\section{Fig. 2}

The valence band (VB) photoemission spectra of Pd on Pt are shown as a function of $\mathrm{Pd}$ coverage. The incident photon energy was set to $90 \mathrm{eV}$, and the experimental geometry is identical to Fig. 1 spectra.

\section{Fig. 3}

The $d$-band centers and widths of valence band (VB) spectra from Fig. 2 are plotted as a function of Pd coverage on Pt surface. 


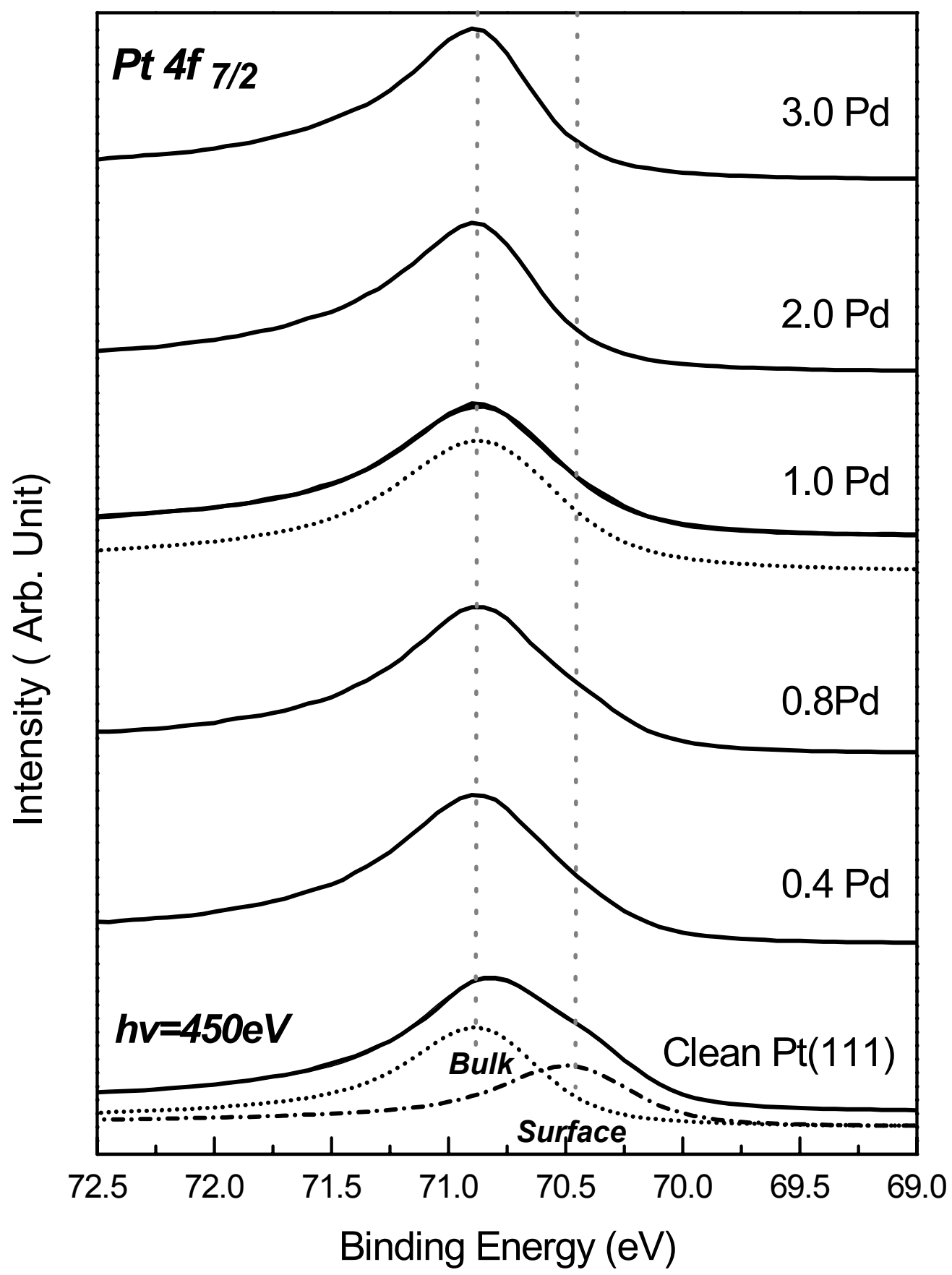

Figure 1(a) 


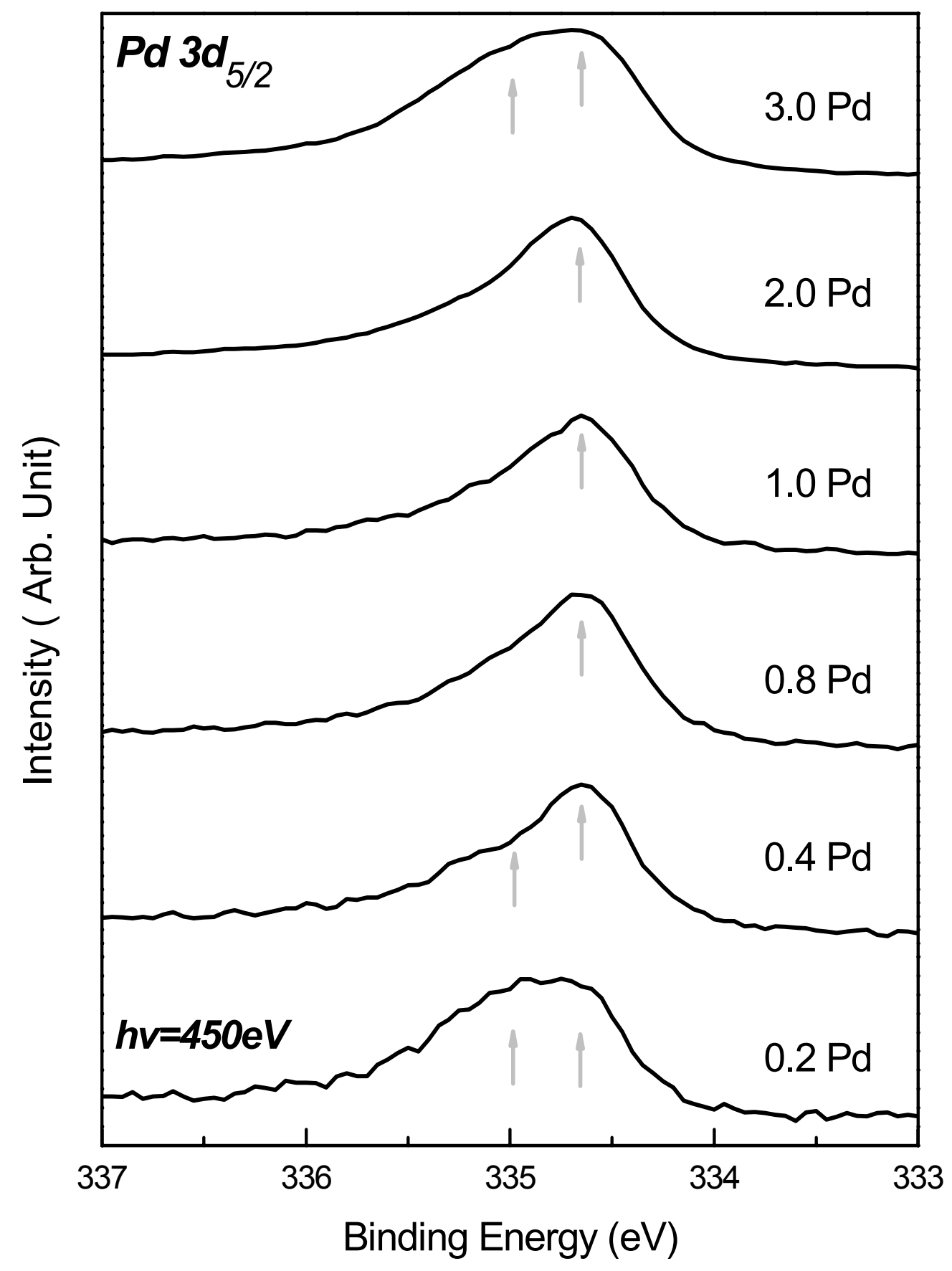

Figure 1(b) 


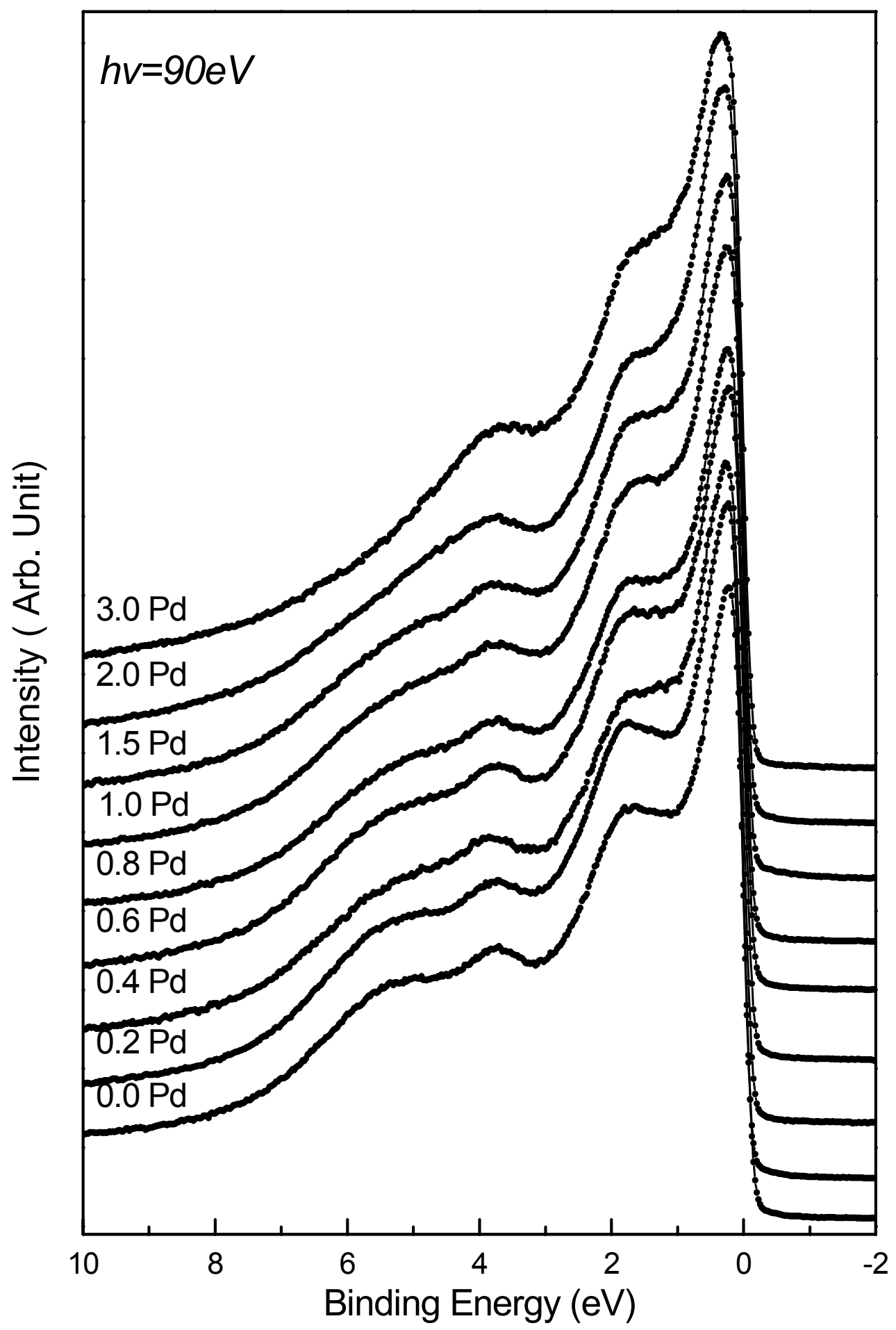

Figure 2 


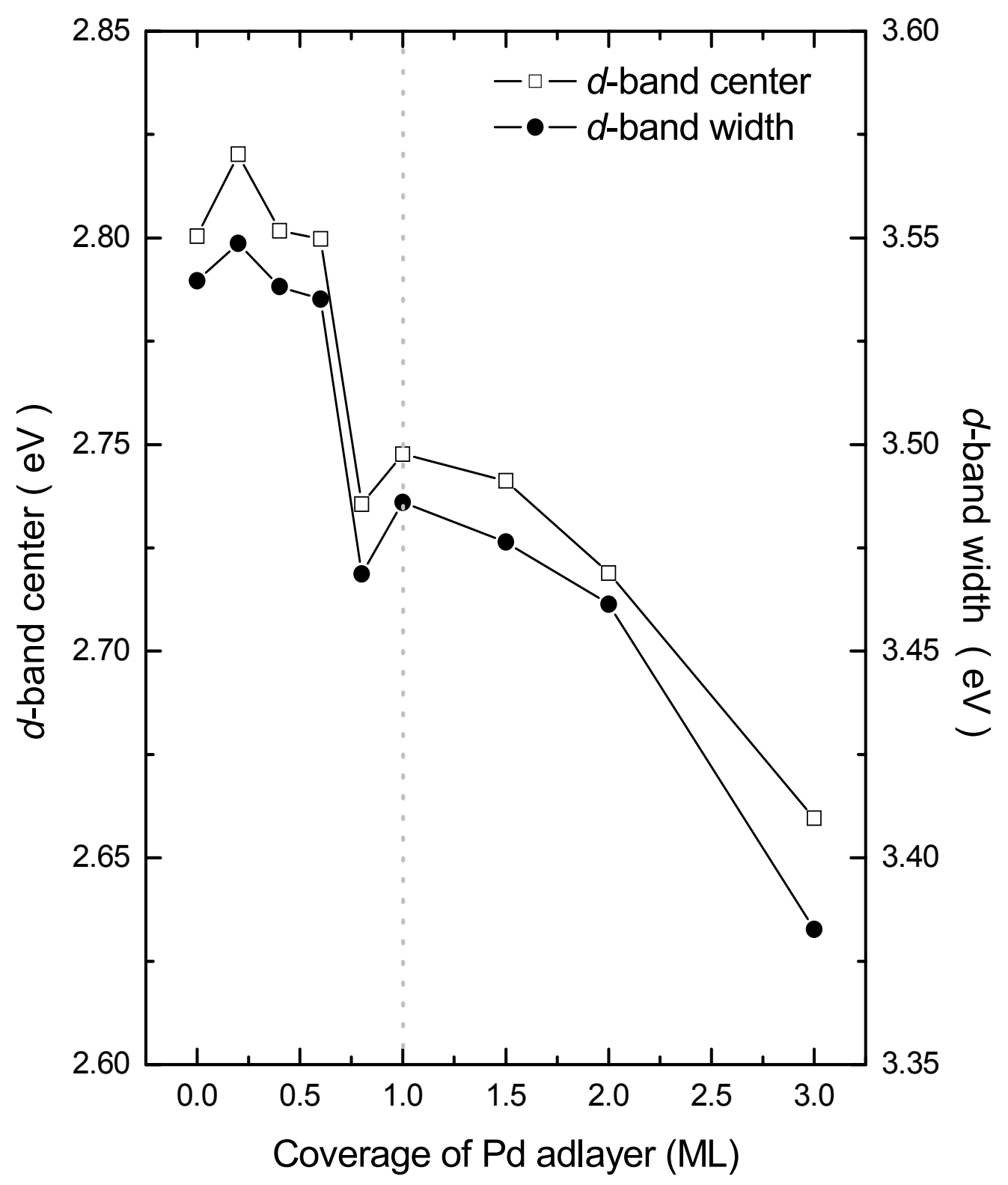

Figure 3 


\section{Reference}

${ }^{1}$ W.F. Engelhoff, Jr., Surf. Sci. Rep. 6. 253 (1987)

2 J.A. Rodriguez, D.W. Goodman, Science 257, 897 (1992) ; R.A. Campbell, J.A.

Rodriguez, and D.W. Goodman, Phys. Rev. B. 467077 (1992)

${ }^{3}$ C.S. Fadley, Electron Spectroscopy : Theory, Techniques, and Applications, C.R.

Brundle and A.D. Baker, eds., Vol. II, Chap 1 ( Academic Press, London, 1978)

${ }^{4}$ P.H. Citrin, G.K. Wertheim, and Y. Baer, Phys. Rev. Lett. 41, 1425 (1978), M. C.

Desjonqueres, D. Spanjaard, Y. Lassailly and C. Guillot, Solid State Commun. 34, 807 (1980)

${ }^{5}$ Ch. Kuhrt and M. Harsdorff, Surf. Sci, 245, 173 (1991); G. K. Wertheim, Z. Phys. D 12, 319 (1989)

${ }^{6}$ N. M. Marković and P. N. Ross, Jr., Surf. Sci. Rep. 45, 121 (2002)

${ }^{7}$ Moonsup Han, P. Mrozek, and A. Wieckowski. Phys. Rev. B. 48, 83291993

${ }^{8}$ Z. Hussain, T. Huff, S.A. Kellar, E. Moler, P.A. Heimann, W.R. McKinney, C.

Cummings, T. Lauritzen, P. McKean, J. Palomares, H. Wu, Y. Zheng, A.T. Young, H.A.

Padmore, C.S. Fadley, and D.A. Shirley, Rev. Sci. Instrum. 69, 9, (1995).

${ }^{9}$ M. J. Ball, C. A. Lucas, N. M. Markovic, V. Stamenkovic and P. N. Ross, Surf. Sci. 518, $201(2002)$

${ }^{10}$ R.Kern, G. Le Ley, and J.J. Metois in Current Topics in Materials Science, edited by Kaldis (North-Holland, Amsterdam, 1979) Vol. 3.

${ }^{11}$ Bongjin Mun, Choongman Lee, Vojislav Stamenkovic, Nenad M. Markovic, Philip N. Ross Jr., in print Phys. Rev. B. 
${ }^{12}$ The position of the center of valence band and its width are defined as $\mu_{1} / \mu_{0}$, where $\mu_{p}=\int N(\varepsilon) \varepsilon^{p} d \varepsilon .(N(\varepsilon)$ is the DOS and $\mathrm{p}$ is is the order of moment.) Throughout the entire calculations of $d$-band centers and widths, the upper limit of integrations on valence band spectra is set to $10.0 \mathrm{eV}$ binding energy position.

${ }^{13}$ N.W.Ashcroft and N.D.Mermin, Solid State Physics (Saunders College Publishing, Philadelphia, 1976)

${ }^{14}$ M. Mavrikakis, B. Hammer, and J. K. Nørskov, Phys. Rev. Lett. 81, 2819 (1998) 